\title{
Strengthening the quality of paediatric primary care: A process evaluation of a complex health systems intervention in South Africa
}

\section{Robyn Leigh Curran ( $\nabla$ robyn.curran@uct.ac.za )}

University of Cape Town Lung Institute https://orcid.org/0000-0003-1337-0011

Jamie Murdoch

University of East Anglia School of Health Sciences

Ruth Vania Cornick

University of Cape Town Lung Institute

\section{Sandra Picken}

University of Cape Town Lung Institute

\section{Max Bachmann}

University of East Anglia Norwich Medical School

\section{Eric Bateman}

University of Cape Town Lung Institute

\section{Makhosazana Lungile Simelane}

University of Cape Town Lung Institute

\section{Lara Rosemary Fairall}

University of Cape Town Lung Institute

\section{Research article}

Keywords: paediatric primary care, PACK, process evaluation, IMCl, health system strengthening

Posted Date: December 13th, 2019

DOI: https://doi.org/10.21203/rs.2.18890/v1

License: (1) (1) This work is licensed under a Creative Commons Attribution 4.0 International License. Read Full License 


\section{Abstract}

BACKGROUND: Innovative strategies like WHO's Integrated Management of Childhood Illness (IMCI) have resulted in progress in addressing infant and child mortality. However, the needs of children continue to present a burden upon primary healthcare services. The capacity of services and quality of care offered require greater support to address these needs and extend integrated curative and preventive care, specifically, for the well child, the child with a long-term health condition and the child older than 5 years, not included in IMCI. In response to these needs, the PACK Child intervention was developed, based on a similar successful approach in adults, that expands the scope and reach of integrated management and training programmes for paediatric primary care. We report findings from the process evaluation of the integration of PACK Child intervention within the existing primary health care system.

METHODS: A mixed methods process evaluation was completed in ten primary health care facilities in the Western Cape province in South Africa, where clinicians were trained to integrate PACK Child into routine practice. Qualitative data included interviews with managers, clinicians, caregivers and policymakers; observations of training, consultations and clinic flow. Quantitative data included training logs and clinicians' questionnaires.

RESULTS: Impact of PACK Child on clinical practice: Longer consultations, improved clinical knowledge and practice, better teamwork, strengthening of appropriate referrals and inclusion of psychosocial risk screening. Implementation fidelity of PACK Child was hindered by over-reliance on documentation and embedded checklist approach to consultations limited to acute episodic illnesses. PACK Child increased caregiver participation during consultations.

CONCLUSIONS: The PACK Child intervention has strengthened primary care for children despite institutionalised care provided by a limited number of clinicians focusing on acute illness with little participation of the caregiver. With refinement of the programme, there is potential for PACK Child to strengthen paediatric care in low-resource settings.

\section{Background}

South Africa has not met the child mortality target for the Millennium Development Goals, in spite of having invested substantially in programmes and policies to achieve these targets.(1) The Sustainable Development Goal targets for child mortality rate and infant mortality by 2030 are $<25$ and $<12$ per 1000 live births, respectively. However, in 2016 these rates were 42 and 35 and their decline has slowed.(2) The World Health Organisation's Integrated Management of Childhood Illness (IMCl) strategy played a role in shaping primary healthcare for children under five in LMICs for the past twenty years and has contributed to the decline in child mortality. Although it has seen many successes,(3-7) implementation was challenged by inadequate local adaptation and infrequent revision of content, insufficient staff training and supervision, variable uptake and inconsistent use in primary care. In addition, IMCl covers priority lifelimiting conditions like diarrhoea, pneumonia, HIV and TB, but does not address other common and 
increasingly pressing problems like asthma, allergies, epilepsy and mental illness. Lacking too is guidance for children over 5 years, management of long-term health conditions, and more thorough integration of curative and preventive measures, including importantly, routine care of the well child.

In the Western Cape province of South Africa almost every public sector primary care facility employs an IMCl-trained nurse, and it is these nurses who attend to the majority of children's healthcare care needs. At a series of meetings with key stakeholders in provincial paediatric health - primary care nurses, doctors, managers and educators, hospital-level paediatricians and policy makers - the growing gaps in knowledge and expertise for children at primary care level were recognised as well as a need to integrate well child routine care into the delivery of everyday paediatric primary care.

This prompted the development of an expanded programme to address a larger remit of paediatric care. Led by the University of Cape Town's Knowledge Translation Unit (KTU), the PACK Child intervention was based on the Practical Approach to Care Kit (PACK) Adult programme that has supported the delivery of comprehensive, integrated adult primary care in the province for the past 13 years. $(8,9)$

Implementation of a health system strengthening intervention like PACK in a health system is a complex activity, requiring an understanding of how it will interact with varying contexts of delivery. The Department of Health was especially keen that we address stakeholder concerns of its integration with existing programmes and policies, particularly $\mathrm{IMCl}$. To explore these issues and address concerns, a process evaluation was conducted alongside a pilot of PACK Child to determine what refinements are needed at intervention and health system levels to optimise its implementation.

The process evaluation provided many rich insights on the tension between contextual features of South African paediatric primary care and how clinicians attempted to integrate the PACK Child intervention within primary care consultations. These are addressed in more detail in another paper providing in-depth understanding of the complexities of paediatric primary care, and the challenges of embedding an intervention within this context.

In this paper, we provide an overview of the process evaluation findings as the PACK Child intervention evolved during the pilot, including how the health system interacted with intervention delivery, and perspectives of how PACK Child impacted clinical practice and the needs of caregivers. In the discussion, we provide suggestions on how to optimise delivery of PACK Child for wide-scale implementation in primary care with consideration of barriers and facilitators to change.

\section{Methods}

\section{Setting}

The pilot and process evaluation took place in 10 public sector primary health care facilities serving impoverished urban and rural communities in the Western Cape, South Africa. Phase One took place in a single facility, Phase Two in an additional three facilities and Phase Three, six facilities. 
The facilities were purposively selected in consultation with the Western Cape Health Department's People Development Centre, which oversees training and upskilling of public sector healthcare workers in the Western Cape. Study sites were chosen to provide maximum variation of primary care delivery across urban and rural communities, [see Additional File 1]. Factors considered important for observing variation included whether clinics were Ideal Clinic sites, (an initiative to improve quality of primary healthcare)(10); number of IMCl-trained nurses; differing levels of PACK Adult training coverage; and use of Integrated Clinical Stationery (an initiative to standardise documentation and facilitate continuity of care of children up to six years).

\section{PACK Child Intervention}

The PACK Child programme comprises a clinical decision support tool (guide), staff training and health systems strengthening elements to enable its use in everyday practice. The guide is a comprehensive, integrated and policy-aligned clinical decision support tool providing an approach to $63 \mathrm{common}$ symptoms, and 16 priority long-term health conditions for children from birth to age 13).(11) It also contains several 'Routine Care' pages [see Additional file 2]-standardised checklists for the provision of well child routine care which covers aspects of nutrition, growth, immunisations, HIV and TB screening, and psychosocial risk of both carer and child. Following the implementation strategy of the PACK Adult programme,(12) PACK Child employs key methods to enable scalability and sustainability. Governmentemployed staff serve as trainers to deliver, via a cascade model, onsite, educational outreach sessions to the clinical team using a training methodology underpinned by adult education principles.(12) The $1 \frac{1}{2}$ to 2-hour training sessions focus on priority content and key messages, using a curriculum of case scenarios to convey these messages and embed use of the guide into everyday clinical practice [see Additional File 3]. Health systems strengthening components include: clarification of prescribing roles among cadres of clinicians, strengthening referral pathways with secondary and tertiary level hospitals through sensitisation training, and assessment of patient clinic flows and referral pathways. A KTU trainer, who has extensive experience with children and has worked both as an $\mathrm{IMCl}$ nurse and trainer (MS), trained clinicians on-site during the first two phases and then trained facility trainers in a five-day workshop to train staff in the remaining six facilities include in Phase Three. Additional file 4 shows the evolution of the intervention across the three phases of the pilot, integrating feedback from the process evaluation and directly from staff at facilities, including the addition of health systems strengthening components.

\section{Design}

We used a linguistic ethnographic methodology, $(13,14)$ which provides theoretical and methodological tools for analysing social action within context. We have previously adapted this approach, to facilitate detailed investigation of complex healthcare interventions across macro-, meso- and micro-contextual levels.(15) Detailed methods are described elsewhere. (16)

The process evaluation used a mixed method approach included quantitative and qualitative data collection methods in all facilities. Qualitative methods included observations of training sessions; semi- 
structured interviews with caregivers; clinician, policymaker and paediatric manager focus groups, and ethnographic observations of consultations and non-clinical areas in each facility. Quantitative methods included auditing of training attendance logs and a clinician' questionnaires completed six months after finishing the PACK Child training programme.

\section{Study population}

Participants included in this study were caregivers, and their children where appropriate; clinicians working at the selected facilities, which included nurses (professional nurses (PN), clinical nurse practitioners (CNP), enrolled nurses (EN), enrolled nursing assistants (ENA)), doctors and pharmacists; PACK Child facility trainers; facility managers, local district structure representatives and paediatric programme co-ordinators and policymakers.

To be eligible for inclusion, nurses and doctors needed to receive PACK Child training and caregivers and children to be receiving paediatric primary care at the selected facilities. Children needed to be aged $0-$ 13 years to receive paediatric services. Policymakers and managers needed to be responsible for delivery of public sector primary care in South Africa.

\section{Sampling}

Data collection for the process evaluation occurred concurrently with the three phases of the pilot, enabling analysis of Phase One data to inform the sampling strategy in Phases Two and Three. Purposive sampling was planned in Phase One to select and recruit managers, clinicians, caregivers and children. Sampling of children in Phase One was intended to be informed by diversity of conditions, level of deprivation and the age of the child. However, in practice, we responded to children presenting on days during which data collection took place, with clinic nurses identifying and approaching caregivers of eligible participants in clinic waiting rooms. Findings from the analysis of Phase One qualitative observation and interview data, (for example, children's presenting conditions, or challenges to using the PACK Child guide), informed theoretical sampling (17) of clinicians, caregivers and children and timing of data collection in Phases Two and Three. In addition, the type of consultation observed in Phase One included mostly children under 5 years presenting with acute symptoms. In Phase Two and Three, we tried to include more consultations with older children and those with long term health conditions, however due to embedded patterns of caregivers/children seeking care, this proved difficult to implement.

We observed all training sessions in Phase One to understand how each session was delivered by trainers and received by clinicians. Sessions in which we identified tensions, or difficulties in delivery were observed in Phases Two and Three such as difficulties in using different documentation and orienting to symptoms as potential markers of long-term health conditions. In Phase One, ethnographic observations of consultations were conducted after each session of the PACK Child training. This highlighted that clinicians needed time to familiarise themselves with the PACK Child guide. In Phases Two and Three we therefore decided to conduct observations of consultations towards the end of the training programme. We invited all managers at facilities to be interviewed and we observed non-clinical areas in all facilities. We conducted a stakeholder workshop with 23 representatives from the pilot facilities, local district 
structure representatives and paediatric programme co-ordinators to present the pilot findings and facilitate discussions on the implications of PACK Child for wider implementation.

\section{Ethical Considerations}

Ethics approval was obtained from University of Cape Town Human Research Ethics Committee, City of Cape Town Research Ethics Committee and the Western Cape Provincial Health Research Committee. The key ethical principles of voluntary and informed participation, confidentiality and safety of participants were used in all researcher and participant interactions. Written consent for interviews and observations was obtained from all facility managers, clinicians and caregivers. Children over seven years old were asked to give assent to their participation. Caregivers and children were asked to consent to interview and observation on the day they attended the clinic. Facility managers provided consent for observations of training sessions and non-clinical areas. All participants were provided with written information about the research, informed that their participation was voluntary and that they could withdraw from participation at any time.

\section{Data analysis}

Qualitative interviews and focus group data, and field notes of our observations of waiting rooms and reception areas were analysed to understand how facilities were organised to provide paediatric primary care, identify contextual features likely to impact on intervention delivery, and to obtain perspectives of the existing primary healthcare system and the function of PACK Child within that system. We also identified which documents clinicians used during paediatric primary care delivery (e.g. IMCl checklist, Integrated Clinical Stationery, PACK Child, other facility documentation) and compared the structure and content of each document. All focus group and interview discussions were transcribed verbatim and thematically analysed, conducting open, then axial coding of transcripts to develop higher order categories. One researcher (RC) completed all the coding of interviews and a second (JM) coded $10 \%$ of the data in the first phase of the pilot. Disagreements in coding categories and choice of coding within the subset were discussed, with coding and coding categories refined as a result. Quantitative survey and training attendance log data were analysed to provide descriptive statistics of intervention delivery across the three phases.

To understand how the context of primary care interacted with delivery of PACK Child we analysed observations of training sessions and consultations. To assess fidelity we drew on MRC guidance that understanding adaptation to the local context is more appropriate than a strict assessment of fidelity.(18) Unlike studies that assessed nurse fidelity to IMCl guidance in a large sample of consultations $(19,20)$ using a fidelity checklist, we chose to incorporate an understanding of the interaction between clinician's use of the guide and the organisational and social context in which PACK Child was being introduced. This required in-depth qualitative investigation in a relatively smaller number $(n=53)$ of consultations and sites. We analysed the audio-recordings, transcriptions and researcher field notes of consultations to understand how contextual features shaped nurse's interactions with caregivers and children.(16) 
We then triangulated all qualitative and quantitative data in order to obtain in-depth insight into the relationship between PACK Child and the context of paediatric primary care, allowing us to generate hypothetical propositions for optimising implementation of the intervention more widely.

\section{Findings}

The findings are presented in three broad areas considering how delivery of PACK Child changed as the intervention evolved across the three phases of the pilot. First, are findings from clinician interviews, triangulated with observations of PACK Child training sessions, of how different intervention components impacted clinical practice. Second, findings of intervention fidelity, drawing on observations of consultations, as well as clinicians' reports of how they used PACK Child in everyday practice. Finally, we report caregiver perspectives of their experience of PACK Child consultations.

The overall sample included four policymakers, 10 managers, 70 clinicians and 69 caregiver/child dyads, one KTU trainer and three trained clinicians delivering training within their facility. We conducted a total of: 10 non-clinical observations, 53 consultation observations, 31 observations of training sessions, 52 caregiver interviews (34 of whom also had their consultations observed), 10 clinicians' focus groups and a stakeholder workshop with 23 participants. Sixty-four clinicians from all pilot clinics completed the clinician's questionnaire six months after completing the PACK Child training sessions, a response rate of $65 \%$. High staff turnover meant many staff who had been trained could not be contacted six months after completion of training (Table 1). 
Table 1

Data collection across all phases of pilot

\begin{tabular}{|c|c|c|c|c|}
\hline $\begin{array}{l}\text { Types of } \\
\text { Data }\end{array}$ & Phase 1 & Phase 2 & Phase 3 & Total \\
\hline Facilities & 1 & 3 & 6 & 10 \\
\hline $\begin{array}{l}\text { Clinicians } \\
\text { trained }\end{array}$ & 15 & 24 & 60 & 99 \\
\hline $\begin{array}{l}\text { Non-Clinical } \\
\text { Observations }\end{array}$ & 1 & 3 & 6 & 10 \\
\hline $\begin{array}{l}\text { Consultation } \\
\text { Observations }\end{array}$ & $17^{*}$ & 12 & 24 & 53 \\
\hline $\begin{array}{l}\text { Training } \\
\text { Observations }\end{array}$ & 8 & 13 & 10 & 31 \\
\hline $\begin{array}{l}\text { Manager } \\
\text { Interviews }\end{array}$ & 1 & 3 & 6 & 10 \\
\hline $\begin{array}{l}\text { Caregiver } \\
\text { Interviews }\end{array}$ & $20 * \star$ & 12 & 20 & 52 \\
\hline $\begin{array}{l}\text { Clinician } \\
\text { Focus } \\
\text { Groups }\end{array}$ & 1 & 3 & 6 & 10 \\
\hline $\begin{array}{l}\text { Clinician } \\
\text { Survey }\end{array}$ & 7 & 17 & 40 & $64 * \star \star$ \\
\hline $\begin{array}{l}\text { Trainer } \\
\text { Interview }\end{array}$ & 1 & 0 & 2 & 3 \\
\hline $\begin{array}{l}\text { Document } \\
\text { analysis }\end{array}$ & $\begin{array}{l}\text { Integratec } \\
\text { (Under } 5 \\
\text { care) } \\
\text { Road to } \\
\text { (Handhel } \\
\text { IMCl chec } \\
\text { (Separate } \\
\text { consultat } \\
\text { or malnut }\end{array}$ & $\begin{array}{l}\text { ionery } \\
\text { dtine care } \\
\text { aregivers } \\
\text { r under } 2 \\
\text { exclusion } \\
\text { ia; HIV/TE }\end{array}$ & $\begin{array}{l}\text { onitoring, i } \\
\text { hild's heal } \\
\text { ns, cough } \\
\text { immunis }\end{array}$ & $\begin{array}{l}\text { S and chronic } \\
\text { years of life) } \\
\text { leted in } \\
\text { ver, ear problem }\end{array}$ \\
\hline $\begin{array}{l}\text { Stakeholder } \\
\text { workshop }\end{array}$ & $\begin{array}{l}\text { Held at er } \\
\text { policymal } \\
\text { and partic }\end{array}$ & $\begin{array}{l}\text { Ine 2019) } \\
\text { s/pilot pa } \\
\text { buted to }\end{array}$ & $\begin{array}{l}\text { holders in } \\
\text { eedback gi } \\
\text { i recomm }\end{array}$ & $\begin{array}{l}\text { ss evaluation } \\
\text { optimisation. }\end{array}$ \\
\hline \multicolumn{5}{|c|}{$\begin{array}{l}\text { * Two of the consultation observations were well child visits. No questions related to the child's health } \\
\text { were asked in these consultations. }\end{array}$} \\
\hline \multicolumn{5}{|c|}{ **In Phase 1 caregiver interviews were conducted throughout the pilot. } \\
\hline
\end{tabular}




\section{The impact of PACK Child on clinical practice}

Overall, the data collected suggested positive impacts on clinical practice, improving clinical knowledge and practice, fostering teamwork, improving referral processes, and caregiver's participation in the consultation process. This was in spite of several difficulties for clinicians in incorporating PACK Child within a practice usually focused on care of acute illness, time limitations and verticalized care with only designated nurses attending children.(21)

\section{Length of consultations}

Analysis of consultation observations suggested that where clinicians followed the PACK Child guide in detail, which included routine care for the well child, consultations were longer than where clinicians only partially used the guide. Other factors appearing to influence consultation length included the number of symptoms the child presented with, previously unidentified problems now identified through the clinician's use of the guide, and staff using PACK Child who did not routinely attend children.

Table 2 shows the average consultation length in Phase Three was 7-8 minutes longer than the other two phases and included an average of 10-13 more questions per consultation. However, there were also a few extreme cases in Phase Three, with one consultation comprising 84 questions and lasting 52 minutes due to the child presenting with multiple problems that required assessment and which the nurse dealt with in turn. 
Table 2

Length of consultations and average number of questions

\begin{tabular}{|llll|}
\hline Phase & \multicolumn{2}{l|}{ Consultation Length (Minutes: Seconds) } & Questions \\
\hline $\begin{array}{l}\text { Phase One } \\
(\mathrm{n}=14)\end{array}$ & Mean & $18: 18$ & 17.21 \\
\cline { 2 - 4 } & Range & $12: 11-33: 34$ & $4-35$ \\
\cline { 2 - 4 } & SD & $5: 55$ & 8.37 \\
\cline { 2 - 4 } & Interquartile Range & $8: 06$ & 12.5 \\
\hline $\begin{array}{l}\text { Phase Two } \\
(n=13)\end{array}$ & Mean & $17: 32$ & 20 \\
\cline { 2 - 4 } & Range & $10: 19-33: 40$ & $4-37$ \\
\cline { 2 - 4 } & SD & $5: 51$ & 9.09 \\
\cline { 2 - 4 } & Interquartile Range & $5: 23$ & 10 \\
\hline $\begin{array}{l}\text { Phase Three } \\
(n=24)\end{array}$ & Mean & $25: 41$ & 30 \\
\cline { 2 - 4 } & Range & $10: 58-52: 21$ & $5-84$ \\
\cline { 2 - 4 } & SD & $10: 21$ & 20.2 \\
\cline { 2 - 4 } & Interquartile Range & $13: 41$ & 18.25 \\
\cline { 2 - 4 } & & & \\
\end{tabular}

Although use of PACK Child appeared to entail longer consultations, some staff reported that increased familiarity with the guide, through regular use over one or two months, would reduce the duration of consultations.

"Sometimes it takes too long, because I work alone in the room, and I have about 30 patients, sometimes more than 30 that I see in a day, then it takes a while, but the more you familiarize yourself with the guideline it will go quicker, because you'll know the questions to ask, but for now it's taking a little bit of time." (Nurse, Focus group, Phase 3)

\section{Improving clinical knowledge and practice}

Eighty-seven per cent of surveyed clinicians reported that the PACK Child guide and training programme improved their ability to both diagnose and treat children even though $85 \%$ of them were already trained in IMCl.

One area where this was especially pronounced was on growth monitoring. One of the training sessions is dedicated to embedding correct monitoring and interpreting growth in children. Using case scenarios, clinicians are asked to plot children's growth measurements on a chart and interpret their growth using the PACK Child guide. Many nurses initially struggled to determine where to plot measurements on growth monitoring charts. After this session, staff reported growing confidence in their ability to plot and interpret the growth of children. 
"And the assessing and interpreting the growth let alone the minor things, plotting the growth chart which we were doing, but now we know how to do it in the right way. But now that we have done PACK Child, we know the way to do and assess whether they are implementing it right. It starts with weigh, measuring and plotting. If you plot wrongly, it will be interpreted wrongly..." (Nurse, Focus group, Phase 2)

PACK Child's expanded scope of content to include long-term health conditions like HIV, tuberculosis, eczema and asthma appeared to have a definite impact on clinicians' management and diagnosis of long-term health conditions and conditions not covered extensively by $\mathrm{IMCl}$, such as skin conditions.

"For example, I can say PACK guideline is very helpful, because I'm going to mention like skin symptoms. I used to see a child with a rash, but I couldn't differentiate what is it really, but when I go to the PACK Child, I know I can name it. It has got its specific diagnosis. I know what it is. But when I look at the PACK Child and I look at the child, then I see exactly what is in the PACK Child, and also what kind of treatment. It's very helpful." (Nurse, Focus group, Phase 3)

"According the PACK guideline it shows you exactly on the X-ray what type of TB it is on those pictures. So you can start(TB treatment) already. Doctor cannot say you were not supposed to start, because you have other things also to back you such as the Mantoux or the weight loss and all those things. So then you can just continue and confirm with the doctor." (Nurse, Focus group, Phase 2)

Several nurses appreciated guidance in PACK Child for children over five years, guidance that was previously limited.

"So, with PACK Child it's much better, you feel more secure that now you can treat the child until 12/13 years old." (Nurse, Focus group, Phase 2)

Many clinicians also reported that the algorithms were clearer in PACK Child than in IMCI despite having the same or similar information.

"What we have noticed is that the algorithm we have got from PACK Child is more clearer than IMCl, although the information is the same or similar, but it is more clearer." (CNP, Phase 2)

However, clinicians experienced some difficulty in shifting the focus of care from an acute episodic paradigm to one of long-term health conditions, especially in the management of recurrent respiratory conditions. Several doctors reported being hesitant in supporting nurses to prescribe inhaled medication, potentially discouraging nurses' from taking advantage of the expanded content of PACK Child.

"In the asthma case presented in the session, the child presented with a recurrent wheeze for five days. The child was given a trial of an inhaler, but the clinicians omitted checking the bronchodilator response before prescribing. The doctor in the training felt that nurses would be prone to abuse inhalers if they were not assessing how previous episodes were managed and the correct diagnostic process followed including checking bronchodilator response." (Field notes, Observations of Session 7: Long Term Health Conditions, Phase 3) 


\section{Fostering teamwork}

A key function of the PACK Child training approach, through the inclusion of all cadres of clinicians, was to encourage collaborative working in order to reduce verticalized delivery of paediatric primary care. Although enrolled nurses and pharmacists reported that much of the training was not directly relevant to their current role, they expressed the value of the training for orientating them to a broader scope of paediatric primary care and the benefits of collaborative working for sharing knowledge. Enrolled nurses also noted that the training helped to delineate the roles and responsibilities of different cadres so that tasks were shared and enabled greater collaboration across clinical staff.

"For me, as a junior nurse, I thought perhaps why am I involved in this, because now I see this book that is being used by the CNPs and the doctors but now as l've gone along, and ((trainer)) brought a triage page which specifies what is it that us junior nurses we need to do, before the child could go to a CNP. I saw it very much helpful. So that the CNP or the clinician doesn't take much time in doing the little things that was supposed to be done by us." (Enrolled Nurse, Focus group, Phase 3 )

The "health system strengthening session", which primarily focused on the flow of children through the clinic, facilitated changes in some facilities. Where verticalization of care was predominant such as one nurse weighing all the children, nurses in consultation rooms began to alleviate the burden of tasks traditionally limited to certain cadres, which promoted task-sharing amongst clinicians.

"We don't need to move the child from your room. You can weigh the child there." (Nurse, Focus Group, Phase 2)

Facilities reported that triaging of children was streamlined, and nurses felt equipped to identify children needing referral within the clinic, which fostered team work and shorter waiting times.

"The other thing for triaging of the patients, the babies they really get emergency care much quicker, also their routine screening is so much easier with the length mat is there, everything is there. So, the staff have really benefitted from the training."(Manager, Phase 2)

\section{Strengthening appropriate referral processes}

Sixty-one per cent of survey respondents reported that use of PACK Child reduced the frequency of referrals, or the need to ask advice from other staff members. This was evidenced in staff focus group discussions.

"What I have noticed is that she is always consulting, she is knowledgeable, almost to her utmost best of what is in PACK. But I have not seen her coming to me with a challenge she cannot go beyond. Even those kids that are referred to the emergency section. Sometimes, she doesn't even come to me. She picks up the problem for the emergency and she send them without my intervention." (Clinical Nurse Practitioner, Focus group, Phase 2) 
Changes in referral patterns reported by staff emphasised increased appropriate referrals for children who were previously under-referred for growth issues or long-term health conditions, and decreased inappropriate referrals for children who were previously over-referred to doctors or tertiary level hospitals for issues which could be safely and effectively managed in primary care.

"I can say I have a lot of children coming with a lot of skin conditions and now I can treat them. Because they come in and say I want to be referred to ((name of hospital)). And I didn't need to refer them, I say let us just try our treatment here, that is why I say unnecessary referrals to tertiary level. So now I am treating them here and they are coming back in a months' time to review the skin." (Nurse, Stakeholders workshop, Phase 3)

During qualitative interviews, nurses reported that use of the screening tools within PACK Child led to them identifying more children with problems that required referral. For this nurse, this was specifically in relation to identifying overweight children requiring referral, utilising PACK Child's guidelines and parameters for children's growth and development.

"We picked up lots of obese babies, of which now we are referring to the dietician and the dietician now has something to do. Before we were only picking up children with malnutrition. We didn't consider the obese, now we know when to refer, we know which weight is expected of each child, so we know when to refer. So now really it is of help." (Nurse, Focus group 2, Phase 2)

In Phase One, despite improved identification of children requiring referral, nurses reported frustration with the lack of feedback from hospitals. To address this, the PACK Child intervention team set up a meeting with the local hospital to orientate the staff there to PACK Child and discuss existing referral challenges. Despite this, poor communication of the outcome of referrals to hospital care persisted. However, in Phase Two PACK Child's standardised approach for referral of children had an impact on how some clinician's felt about referring to secondary care, describing that they felt more able to communicate effectively with secondary care clinicians.

"Even if we phone a doctor to refer a child at the hospital, you know exactly what you're talking about. It's not like you question yourself, as it is in the guide." (Nurse, Focus group 3, Phase 2)

\section{Improvements in psychosocial risk screening}

According to clinicians, use of PACK Child in consultations led to more psychosocial risk issues being identified in consultations, resulting in some referral and resolution of these disclosures.

"It prompted you now with that section to ask for social problems. I also had one child: she didn't have an ID. Mum didn't have an ID that's why she didn't register the child, and she can't apply for a grant, and I helped her. So that section is really good. It prompts you to ask those questions. In the past we overlooked it." (Nurse, Focus Group, Phase 2) 
However, introduction of routine psychosocial risk questions, in an embedded checklist approach to consultations, appears to result in nurses asking the questions in such a way that limit disclosure of psychosocial problems. Due to the volume of questions to be covered in a consultation, questions are framed in a way to rule out problems instead of encouraging disclosure.(21)

Furthermore, in some cases, where disclosures were made, the clinician could be seen to minimise its importance or not address the disclosures made by caregivers. This excerpt describes the lack of human resources and one of the reasons why issues that were disclosed may have been minimised.

"We have a problem with psychiatrist. If you get the problem of abuse, then you must send the child to the hospital, because we don't have a person here every day, that's also a problem. Sometimes when you book the people, for that then the guy cancels his visit." (Nurse, Focus Group, Phase 3)

\section{Implementation fidelity of PACK Child}

The PACK Child training encouraged clinicians to approach a consultation in a standard way using the PACK Child guide, starting with an assessment for urgent symptoms and in their absence proceeding to provide well child routine care. Clinicians were trained to navigate the guide for the relevant symptom/s, or long-term health conditions (LTHC) only once they had completed routine care activities.

Our findings revealed several contextual issues which interacted with clinicians' use of the PACK Child guide, affecting fidelity to the training programme's instructions around guide use within consultations. These included how clinicians articulated their use of the PACK Child guide alongside existing documentation and reporting requirements, the approach to guide use (particularly around well child routine care) and the aspirational expectations of what care could be delivered during a consultation.

\section{Navigating PACK Child within current routine practice}

Implementation of the PACK Child approach during consultations was variable and each facility's requirement to use multiple documents resulted in clinicians alternating between different documents and consultation activities. Table 3 illustrates how the PACK Child approach to consultations was interrupted as clinicians switched between different routine care and symptom-based activities and various sections of the guide, whilst also completing necessary documentation. In particular, clinicians typically struggled to follow the consultation sequence recommended by PACK Child guide and training.

Table 3. Activity coding across phases of consultation structure 
The table presents the sequence of activities for one typical consultation within each phase of the pilot. Each consultation is presented in a column. Activities within each consultation are coded according to routine care (italics) and assessment of symptoms (bold), in the sequence they occurred from top to bottom and a description included of the type of activity taking place. Within each activity, the documentation the nurse was using is noted, namely the PACK Child guide (PC), $I \mathrm{MCl}$ checklist (IMCI) and the Road to Health Booklet (RtHB).

\begin{tabular}{|c|c|c|}
\hline PHASE ONE & PHASE TWO & PHASE THREE \\
\hline 14-week-old with conjunctivitis & $\begin{array}{l}\text { 21-month-old with fever, loss of } \\
\text { appetite and painful mouth }\end{array}$ & 4-year-old with cough \\
\hline Elicitation of Problem & Elicitation of problem (PC) & Elicitation of Problem \\
\hline Growth Assessment (RTHB) & Questions about treatment & Symptoms Assessment (IMCI) \\
\hline Symptoms Assessment (RTHB) & Symptoms Assessment (PC) & Questions about Feeding (PC) \\
\hline $\begin{array}{c}\text { Questions about } \\
\text { Immunizations (RTHB) }\end{array}$ & $\begin{array}{c}\text { Physical Exam } \\
\text { (PC-Nurse examines child } \\
\text { mouth/throat) }\end{array}$ & Growth Assessment (PC/RTHB) \\
\hline Developmental Assessment (PC) & Psychosocial Risk (PC) & $\begin{array}{c}\text { Discussion about } \\
\text { other medical problems (PC) }\end{array}$ \\
\hline Growth Assessment (PC) & Questions about Contraception (PC) & $\begin{array}{c}\text { Questions about } \\
\text { Immunizations ( } P C \text { and } R T H B)\end{array}$ \\
\hline $\begin{array}{c}\text { Questions about } \\
\text { Immunizations (RTHB) }\end{array}$ & Developmental Assessment (PC) & Developmental Assessment (PC) \\
\hline Growth Assessment (PC) & $\begin{array}{c}\text { Discussion about } \\
\text { other medical problems (PC) }\end{array}$ & $\begin{array}{c}\text { Discussion about } \\
\text { other medical problems (PC) }\end{array}$ \\
\hline $\begin{array}{c}\text { Discussing other } \\
\text { medical problems (IMCl) }\end{array}$ & Psychosocial Risk (PC) & Symptoms Assessment (PC) \\
\hline Questions about Contraception (PC) & Advice Giving $(P C)$ & Psychosocial Risk (PC) \\
\hline Psychosocial Risk (PC) & Questions about Feeding (PC) & Advice Giving $(P C)$ \\
\hline Symptoms Assessment (IMCI) & Growth Assessment (PC) & Symptoms Assessment (PC) \\
\hline Questions about feeding ( $/ \mathrm{MCl})$ & Nurses Opinion of problem & Physical Exam \\
\hline Symptoms Assessment (IMCI) & Advice Giving $(P C)$ & Advice Giving (PC) \\
\hline Advice Giving (PC) & Questions about Feeding (PC) & Questions about Treatment \\
\hline $\begin{array}{c}\text { Discussion about } \\
\text { other medical problems }(\mathrm{IMCl})\end{array}$ & Physical Exam & Nurses Opinion of problem (PC) \\
\hline \multirow[t]{6}{*}{ Advice Giving } & Symptoms Assessment (PC) & Questions about Treatment \\
\hline & Advice Giving $(P C)$ & Advice Giving (PC) \\
\hline & $\begin{array}{c}\text { Discussion about } \\
\text { other medical problems (PC) }\end{array}$ & Symptoms Assessment (PC) \\
\hline & Advice Giving $(P C)$ & Advice Giving (PC) \\
\hline & & Symptoms Assessment (PC) \\
\hline & & Advice Giving (PC) \\
\hline
\end{tabular}

Clinicians were able to reflect on the complexity of following different documentation and reported feeling torn.

"Because our mind is now still divided. I've the PACK, I've got the IMCl, you understand? Sometimes if you go now to say, ok, I think I'm more comfortable with the PACK style. Now you more comfortable rather on this, then you mind goes that way." (Nurse Interview, Phase 1) 
A key finding from this work was that the consultation sequence (assess symptom urgency-well child routine care-symptom management) proposed by PACK Child differs from that of the IMCl checklist, requiring clinicians to embrace a new consultation flow. This was especially apparent in Phase One, when the IMCl checklist stationery was used consistently within consultations. Clinicians frequently switched between PACK Child, the IMCl checklist and Road to Health Booklet, often repeating questions that appeared in these documents. This switching between documents, and between routine care and symptom-based activities indicated that staff struggled to navigate between documents and manage the volume and range of tasks required to use PACK Child to attend to both the children's symptoms and their need for well child routine care. In response to these findings, the PACK Child training included two additional components in Phases Two and Three. Firstly, a "health system strengthening session" was included which asked clinicians to map the flow of patients within their facility. This enabled clinicians to consider how to reorganise and reallocate tasks in order to relieve the burden on clinicians in the consultation rooms. Secondly, additional elements were added to an existing session which focused on how best to integrate PACK Child alongside documentation.

Despite introducing the health systems strengthening training session, Phases Two and Three facilities did not make any significant changes to how they organised patient flow and allocation of tasks across clinicians. However, in Phase Two, two of three facilities used blank clinical continuation notes to document findings instead of the IMCI checklist. In Phase Three, whilst there was a mixture of documentation in use, (PACK Child guide, IMCl checklist, Integrated Clinical Stationery) a larger proportion of children above six years (for whom no formal documentation exists) meant that no specific stationery was used within these consultations. Within these consultations, we unsurprisingly identified less switching between documents. However, in both Phases Two and Three we still observed clinicians frequently switching between activities within the PACK Child guide (e.g. assessment of urgency, symptom management, routine care), sometimes doing so before completing an activity, necessitating returning later to complete it.

\section{Following the PACK Child guide as a script}

The distribution of question types shown in Table 4 provides insight into how elements of PACK Child were incorporated into consultations across the three phases, within the embedded IMCl checklist approach. Whilst in Phase Three we still observed switching across activities within the PACK Child guide, analysis of clinician's questioning within consultations suggested that clinicians in Phase Three more closely followed and completed each activity of the guide as proposed within the training sessions.

In all phases the top four question types were oriented to acute symptom management [see Additional file 5], growth monitoring, immunisations, and TB and HIV characteristic of IMCl despite the expanded scope of PACK Child. In Phase Three there were an average of 7.6 questions focused on wider information gathering compared with 2.1 questions in Phase One and 2.9 in Phase Two. These differences may indicate closer fidelity to PACK Child symptom algorithms in Phase Three, but might also reflect the nature of children's individual problems within each phase. However, an average of 4.4 
psychosocial questions per consultation were also asked in Phase Three compared with 1.4 and 3.6 questions for Phases One and Two respectively indicating closer fidelity to routine care questions.

From our direct observations within consultations, we also identified instances of nurses meticulously following every element of the guide in Phase Three, which closely reflected how facility trainers were trained in Phase Three, with emphasis to follow every relevant line of the PACK Child guide within a consultation.

"The training it teaches us that you actually should read to the end, but here now you just grab important things that you catch, but by right we actually supposed to read the whole thing in order to go to the next step." (Nurse, Focus group, Phase 3)

\section{Integrating well child routine care into the consultation}

The identification of clinician difficulty in following the routine care-symptom consultation sequence prompted an important analytical issue: How does asking routine care questions impact on the management of a child's symptoms? We identified that, on average, more symptom questions (reported complaint and wider information gathering combined) were asked when the Routine Care page was not used compared with when it was [see Additional file 6]. It is important to note that this is any use of the Routine Care page, not necessarily complete coverage of all items listed on the page. In many instances nurses only partially used the Routine Care page, missing out several questions, typically around sensitive psychosocial issues.

"I think it is a time thing, the Routine Care goes through quite a lot of psychosocial questions and I suppose those are the ones that I probably leave out." (Nurse, Focus group, Phase 3 )

When the Routine Care page was used, nurses were sometimes not returning to the child's reported complaint following routine care, leaving the complaint unaddressed, or not resolved according to PACK Child recommendations. This demonstrates the difficulty clinicians had in attending to both routine care and the presenting symptoms, with some clinicians reporting a tendency to forget the presenting symptoms.

"You ask symptoms, it may be related to the skin and no urgent signs whatever the case may be and then we have to go routine care, and then we need to note we have to go back and then continue from there, which is where Sister picked up the weight and things which was more alarming because her initial thing that was noted. So that is where Sister also forgot about the symptoms, which was the skin. So, the Routine Care page is a big help, it is a big help, but it is just the back and forth as Sister say." (Nurse focus group, Phase 3)

\section{The impact of PACK Child on caregiver/child participation in care}


Caregivers frequently shared past experiences of bringing their children to the clinic for consultations where the primary focus of care was restricted to the acute presentation.

"But with them if I say his ears are sore, they won't check anything else." (Caregiver Interview, Phase 1)

"Normally the doctors just look. They don't ask a lot of questions. They just look and then they tell you, no it's not like that and it's not like that. The child's fine, you can go." (Caregiver Interview, Phase 3 )

The focus on acute symptoms was identified by caregivers as the core element of most paediatric primary care consultations. Although sometimes attending with recurrent symptoms, caregivers reported that clinicians rarely asked about the child's previous history or referred to documentation, instead treating each episode separately.

"Interviewer: And when you come for the same problem, do they check that she had it before, or do they just treat it like it's new?

Caregiver: They never do, they never like look on her records before, normally she does have tonsils, then they give tablets or something."(Caregiver Interview, Phase 3)

The caregiver view that nurses do not investigate the child's medical history, lack the knowledge to diagnose and adequately treat their child, and that the caregiver feels uninformed.

"Interviewer: Have you brought him to the clinic for the tight chest.

Caregiver: I have come many times

Interviewer: Have they given him anything?

Caregiver: They give an inhaler. The chest is too tight.

Interviewer: Have they said what it is?

Caregiver: They don't know, it's like they don't know, and I don't know.

Interviewer: Did they refer you to anybody.

Caregiver: No, they don't. They don't want to check what is wrong, he is still using the inhaler." (Caregiver Interview, Phase 1)

However, interviews with caregivers emphasised the impact of PACK Child's broadened scope of content on the clinical assessment process. They, frequently reported a marked shift in the questions they were asked in PACK Child consultations compared with that prior to the pilot, which they linked to the clinician's use of the PACK Child guide. 
"It has changed because there was not that book, like there were not those kinds of questions because the last time I was here, I also came for the same problem, but it was not the same he has sore wounds, like there was a wound here, that I came for, but there was not this book asking such questions, they didn't ask so many questions, like they just gave me medication." (Caregiver Interview, Phase 3)

Caregivers also reported that they were not routinely given scheduled visits for their children's long term health conditions, but with PACK Child an appointment was given.

"I just bring (her) in when I see that the nebulizer at home isn't helping, but now the sister gave me threemonth supply with the medication, and she gave me an appointment for after the 3 months; to see us which didn't happen previously." (Caregiver, Phase 3)

Caregivers often conceptualised quality of care in terms of their level of participation in the consultation. Many caregivers reported that when the PACK Child guide was used there was opportunity for them to explain their story, clinicians explained what was happening and that they felt central to the decisionmaking process. This excerpt illustrates the contrast with caregivers' experience of previous visits to the clinic:

"Because it's the third time. Sometimes you go to the doctor then the doctor says, just that one thing. Like this was now nice. Everything was asked, and they have the patience to explain everything. And feel free to explain everything. Sometimes you go to the doctor, you just get cut you off because they rush you to get to another patient. Then that happens all the time." (Caregiver Interview, Phase 3)

Caregivers valued being given the space and time to talk and ask things that were important to them, a sentiment frequently expressed following PACK Child consultations.

"And it was something good that l've learnt today, and I hope they keep it this way; to ask all those questions, and they took their time with us. It was not like before, where the time was really short." (Caregiver Interview, Phase 3)

"Yeah, the way the doctor handled it. It was nice for me, because just for the fact that I can talk a lot of things ask lot of things. He come for his nose, but I could ask for this... she saw the marks of the eczema, almost like eczema." (Caregiver Interview, Phase 3)

Explanation was defined as key to participatory care by caregivers, without the caregiver needing to ask for information. Caregivers described being unable to question nurses when they did not understand the jargon they used. In order to foster participation of caregivers in consultations, using language which caregivers understand is essential, and caregivers stated this occurred when PACK Child was used.

"The only difference is, like now with this consultation. She explained to me properly. A lot of the time the sisters were doing stuff, or give you something, but they don't explain to you what is going on. You must always ask, and you feel so stupid sometimes. You must always ask to explain something to me properly, because you don't know the jargon. So now you have to ask every detail and stuff like that. And 
sometimes they will look at you like you silly, and so that's the only difference, that they explain to you stuff." (Caregiver Interview, Phase 3)

Although many caregivers reported improvements in their level of participation in consultations, this was not reflected in how several clinicians viewed the impact of PACK Child on the clinician-caregiver interaction. They perceived the demands of completing documentation as a barrier that limited their ability to focus their attention on the caregiver and child.

"If you look at the paperwork, you write down on your IMCI form, you have to write down on your clinical as well, you have to write your script. We supposed to write in the child's book, that we must be honest that is not done. You see how many different documents we need to write on. On top that, you starting to mix your medication, you are starting do all this. If you look at it, you more busy writing. You more focusing on writing instead of focusing on the child. (Nurse Interview, Phase 1)

\section{Discussion}

The PACK Child intervention was developed to address the limited scope of $\mathrm{IMCl}$, by expanding a focus in the provision of paediatric primary care from under-fives to children aged up to 13 years and those living with long term health conditions.(11) However, this study illustrates the challenges of implementing PACK Child within a primary healthcare system that currently focuses on acute symptoms in a verticalized pathway of care, with severe time limitations, and in consultations that are often driven by complex and multiple demands for document completion, which reduces meaningful interaction with the caregiver/child. These challenges need to be addressed in seeking to provide care for acute and chronic conditions of children that is comprehensive and person-centred rather than nurse and documentation centric.

Despite these limitations, positive impacts of the PACK Child intervention were reported and observed in clinical practice. Clinicians stated improvements in their clinical knowledge and practice in the diagnosis and management of children, even though most respondents were $\mathrm{IMCl}$ trained. Clinicians specifically mentioned the impact of PACK Child on growth monitoring and interpretation which resulted in increased identification of children with under and over nutrition. PACK Child also expanded the knowledge of clinicians to identify and monitor long term health conditions, diagnose skin conditions and manage children above five years of age, where previously guidance was limited.

Implementation of routine care for all children also led to clinicians asking caregivers about psychosocial issues, and although such questions were often phrased to rule out problems rather than invite disclosure, some psychosocial risks were identified and addressed. We have reported detailed analysis of these interactions elsewhere.(21) These highlight the need for clear referral pathways, consideration of who is best placed to screen for sensitive psychosocial risks within low-resource settings, and training providers to elicit and manage disclosures with legal responsibilities. 
Other clinical practice effects included the role of the PACK Child intervention in fostering team work amongst clinicians at each facility. The inclusive approach of PACK Child to train all cadres of staff differs from the IMCl approach, which focuses on specific cadres of nurse. Our findings confirm the benefit of PACK Child in encouraging collaboration of clinicians, delineating and clarifying clinician's roles and streamlining the triage and patient flow process at facilities.

Whilst our interview and non-clinical observation data do not provide the basis for objectively judging the appropriateness of referrals between cadres, clinicians and managers across facilities reported that PACK Child strengthened referrals within their facility and to other secondary or tertiary level facilities. Where previously under-referral was common, PACK Child resulted in identification of conditions missed in past visits and appropriate referral. For conditions where over-referral was common, PACK Child served to support and provide confidence that these conditions did not require referral and could be managed at primary care.

Unsurprisingly clinicians perceived an increase in consultation length as a consequence of introducing new routine care questions. While staff felt that with practice and consistent use of the guide, consultation times would shorten; they experienced difficulty following the guide meticulously within a time-pressured consultation. Both our observations and interviews revealed that clinicians occasionally forgot to return to the presenting symptom after providing routine care. This was identified as a potential harm and calls into question the need for and feasibility of routine care questions, and whether these can be reduced or tailored to individual needs or included in a different way.

Another barrier to fidelity to the PACK Child intervention was institutionalised facility documentation which, are intended to guide the consultation. PACK Child, with its scripts, conditional statements and, algorithms for care, functions in the same way as this documentation, and resulted in nurses frequently switching between documents and activities while navigating through the guide. A session to streamline the integration of documentation with PACK Child was added and reportedly assisted staff with navigating the guide and existing documents.

Caregivers in the sample emphasised that the current focus of paediatric primary care is persistently on the primary presenting symptom, with poor reference to past medical history. This results in repeated visits for the same symptom. These perspectives aligned with our observations of consultations and echo findings from evaluations of IMCl. In the Bangladesh Multi-country Evaluation of IMCl, only $18 \%$ of consultations assessed all the main symptoms in the child. In studies of clinical assessments of children under-five in Ghana, adherence to all required IMCI tasks occurred in only $1 \%$ of children and adherence to $\mathrm{IMCl}$ case assessment guidelines tasks in only $28.4 \% .(22-24)$ Clinicians in our study confirmed caregiver perspectives that nurses focused on acute symptoms and preferred to conduct IMCl from memory or according to a checklist. Similar findings were reported from a study of healthcare worker adherence to IMCl in Tanzania.(25)

Caregivers' reported perceiving care as being non-participatory, and positioning themselves as passive recipients of care. This, resonates with studies of $\mathrm{IMCl}$ in South Africa, where IMCl implementation 
showed limited caregiver knowledge regarding medication, or when to return to the facility.(19) These findings highlight how caregivers conceptualise quality care, and in our study caregivers also articulated how participation was facilitated through jargon-free guidance when clinicians used PACK Child. This is substantiated in studies on caregivers' perspectives of care where the lack of communication is considered a barrier to person-centred care.(26) In contrast, clinicians' perspectives of care quality juxtaposed those of caregivers, stating that consultations were dominated by requirements to follow different documentation, and PACK Child added another layer to this situation, making it difficult to focus on the child.

Given the continent-wide institutionalisation of $\mathrm{IMCl}$, these insights from our study of pilot facilities in the Western Cape may apply more widely. Although nurses hardly refer to $\mathrm{IMCl}$ guidelines, it is embedded in memory and frames the way in which children are seen and what is prioritised. This may have unwanted effects and unknowingly impact on what is prioritised in a consultation. The demands for completion of documentation appears to be a strong driver and shifts the focus from the caregiver and the child. Only through addressing these tensions is optimal implementation of PACK Child likely and any other programmes that aim to improve the quality of paediatric primary care.

Table 5

Recommendations for optimisation of PACK Child

\section{Recommendations for refinements to the PACK Child guide}

- Improve alignment of PACK Child guide with provincial documentation.

- Prioritise requirements of a well child routine check to make this activity more efficient and feasible, allowing sufficient time to treat symptoms.

- Consider guide revisions to support the delivery of patient-centred care e.g. sequencing and phrasing of questions related to psychosocial risk.

Recommendations for refinements to the PACK Child training approach

- Include referral hospitals in PACK Child training or orientation sessions.

- PACK Child should include how to align PACK Child with IMCI documentation.

- Include a focus on a revised approach to integrating routine care alongside the management of symptoms

Recommendations for health system improvements

- Conduct implementation workshops at district and sub-district level to prepare the health system and ensure managerial buy-in, to discuss issues like stationery, patient flow, and referral pathways to hospitals.

- Clinics need to evaluate patient flow and reallocate acute and routine care tasks to minimise duplication and overburdening nurses.

\section{Strength and Limitations}

This process evaluation was the first study in LMICs to conduct qualitative observations of consultations in paediatric primary care that applied conversational linguistic analysis (21) to audio recordings which 
were then triangulated with observed use of documentation and interviews with clinicians and caregivers. Whilst our findings do not offer statistical generalisability, this in-depth analysis functioned to provide critical insights on the current state of paediatric primary care in the Western Cape, South Africa in a low income setting and the generation of hypothetical propositions for optimising intervention delivery.

Caregiver interviews were often conducted in facility waiting rooms either before or following the child's consultation, which limited the ability to have extended confidential discussions with caregivers.

Sampling of caregivers and children was also limited by those who attended on the day, which may have restricted the broader view of other caregivers who may have attended on different days and attended the clinic regularly. Our findings are also limited by needing to collect data both during and immediately following completion of the PACK Child training programme, which allowed little time for the intervention to be embedded into everyday practice. Whilst our observations of clinicians using PACK Child may have been subject to the Hawthorne effect,(27) arguably affecting the extent to which clinicians used the PACK Child guide, the researcher's presence was less likely to affect how clinicians asked questions and the sequence to the consultation that they delivered.

\section{Conclusions}

In this study, despite many barriers to optimal care, the PACK Child intervention had a positive impact on the delivery of paediatric primary care. It expanded the scope offered, fostered team work and improved the patient/caregiver experience. With further refinement of the programme at a guide, training and system level there is potential for PACK Child to streamline paediatric care in health resource scarce settings globally.

\section{List Of Abbreviations}

Clinical Nurse Practitioner (CNP); Enrolled Nurse (EN); Enrolled Nursing Assistant (ENA); Human Immunodeficiency Virus (HIV); Integrated Clinical Stationery (ICS) ; Integrated Management of Childhood Illness (IMCl); Knowledge Translation Unit (KTU); Lower-middle-income country (LMIC); Practical Approach to Care Kit (PACK); Professional Nurse (PN); Road to Health Booklet (RtHB); Tuberculosis (TB); World Health Organisation (WHO)

\section{Declarations}

Ethics Approval and Consent: Approval was obtained from University of Cape Town Human Research Ethics Committee (568/2017), City of Cape Town Research Ethics Committee (7876) and the Western Cape Provincial Health Research Committee (WC_201709_011). Research participants provided informed consent before any data collection commenced.

Consent for Publication: All participants have consented for the findings of the study to be published with their identity anonymised. 
Availability of data and materials: The datasets generated and/or analysed during the current study are not publicly available due to data transcripts including personal participant information not suitable for sharing, but are available from the corresponding author on reasonable request.

Competing interests: We have read and understood BMJ policy on declaration of interests and declare that Lara Fairall, Eric Bateman, Robyn Curran, Makhosazana Lungile, and Sandra Picken are employees of the KTU. Professor Bateman reports personal fees from Novartis, Menarini, ALK, Sanofi Regeneron, Boehringer Ingelheim and AstraZeneca, for work outside the submitted work. Professor Bateman is also a Member of Global Initiative for Asthma Board and Science Committee.

Since August 2015 the KTU and BMJ have been engaged in a non-profit partnership to provide continuous evidence updates for PACK, expand PACK related supported services to countries and organisations as requested, and where appropriate license PACK content. The KTU and BMJ co-fund core positions, including a PACK Global Development Director, and receive no profits from the partnership. PACK receives no funding from the pharmaceutical industry.

Funding: This research is funded by the Joint Health Systems Research Initiative (Department For International Development, Economic and Social Research Council, UK Medical Research Council and Wellcome Trust). Grant ref: MR/R004080/1. Lara Fairall acknowledges funding from the UK's National Institute of Health Research (NIHR) through King's College London's ASSET Programme on Health Systems Strengthening in sub-Saharan Africa (16/136/54).

Authors Contributions: All authors contributed to the conceptualisation of the research and contributed to writing the manuscript. LF, EB, RVC, MB, JM and RC designed the process evaluation protocol. MLS and SCP led the development of the PACK Child training intervention. RVC led the development of the content of the PACK Child guide. JM and RC collected, analysed and interpretation of all data. RC drafted the manuscript and all co-authors edited and commented on revised drafts. All authors approved the final draft for submission. All authors agree to be accountable for all aspects of the work in ensuring that questions related to the accuracy or integrity of any part of the work are appropriately investigated and resolved.

Acknowledgements: We would like to thank all caregivers and children, clinicians, managers, policymakers and trainers who participated in the process evaluation. The University of Cape Town Lung Institute and Children's Hospital Trust, South Africa provided funding to support the development of the PACK Child guide.

\section{References}

1. Mathews S, Martin LJ, Coetzee D, Scott C, Brijmohun Y. Child deaths in South Africa: Lessons from the child death review pilot. South African Medical Journal 2016;106:851-2.

2. Bamford L, McKerrow N, Barron P, Aung Y. Child mortality in South Africa: Fewer deaths, but better data are needed. South African Medical Journal 2018;108(3):25-32. 
3. Bryce J, Victora CG, Habicht JP, Black RE, Scherpbier RW, Advisors M-IT. Programmatic pathways to child survival: results of a multi-country evaluation of Integrated Management of Childhood Illness. Health Policy Plan. 2005;20 Suppl 1:i5-i17.

4. Chopra M, Binkin NJ, Mason E, Wolfheim C. Integrated management of childhood illness: what have we learned and how can it be improved? Archives of disease in childhood. 2012;97(4):350-4.

5. Gera T, Shah D, Garner P, Richardson M, Sachdev HS. Integrated management of childhood illness (IMCI) strategy for children under five. The Cochrane database of systematic reviews. 2016(6):Cd010123. 6. Costello A, Dalglish S. Towards a grand convergence for child survival and health: a strategic review of options for the future building on lessons learnt from IMNCl. 2016.

7. Fick C. Twenty years of $\mathrm{IMCl}$ implementation in South Africa: accelerating impact for the next decade. South African Health Review. 2017;2017(1):207-14.

8. Fairall L BE, Cornick R, Faris G, Timmerman V, Folb N, et al. Innovating to improve primary care in less developed countries: towards a global model. BMJ innovations. 2015;1(4):196-203.

9. Fairall L, Cornick R, Bateman E. Empowering frontline providers to deliver universal primary healthcare using the Practical Approach to Care Kit. Bmj. 2018;363:k4451.

10. Fryatt R, Hunter J. The ideal Clinic in South Africa: planning for implementation. South African health review. 2014;15:23-43.

11. Picken S, Hannington J, Fairall L, Doherty T, Bateman E, Richards M, et al. PACK Child: the development of a practical guide to extend the scope of integrated primary care for children and young adolescents. BMJ global health. 2018;3(Suppl 5):e000957.

12. Simelane ML, Georgeu-Pepper D, Ras C-J, Anderson L, Pascoe M, Faris G, et al. The Practical Approach to Care Kit (PACK) training programme: scaling up and sustaining support for health workers to improve primary care. BMJ global health. 2018;3(Suppl 5):e001124.

13. Sealey A. Linguistic ethnography in realist perspective 1. Journal of Sociolinguistics. 2007;11(5):64160 .

14. Silva MJ, Breuer E, Lee L, Asher L, Chowdhary N, Lund C. Theory of Change: a theory-driven approach to enhance the Medical Research Council's framework for complex interventions. Trials. 2014;15.

15. Bronfenbrenner U. The ecology of human development: Harvard university press; 1979.

16. Murdoch J, Curran R, Bachmann M, Bateman E, Cornick RV, Doherty T, et al. Strengthening the quality of paediatric primary care: protocol for the process evaluation of a health systems intervention in South Africa. BMJ global health. 2018;3(Suppl 5):e000945.

17. Belgrave LL, Seide K. Grounded Theory Methodology: Principles and Practices. Handbook of Research Methods in Health Social Sciences. 2018:1-18.

18. Moore GF, Audrey S, Barker M, Bond L, Bonell C, Hardeman W, et al. Process evaluation of complex interventions: Medical Research Council guidance. BMJ. 2015;350:h1258.

19. Chopra M, Patel S, Cloete K, Sanders D, Peterson S. Effect of an IMCl intervention on quality of care across four districts in Cape Town, South Africa. Archives of disease in childhood. 2005;90(4):397-401. 20. Thandrayen K, Saloojee H. Quality of care offered to children attending primary health care clinics in Johannesburg. South African Journal of Child Health. 2010;4(3):73-7.

21. Murdoch J, Curran R, Cornick R, Picken SC, Bachmann M, Bateman E, et al. Strengthening the quality 
and scope of paediatric primary care in South Africa: Evaluating contextual impacts of the introduction of the Practical Approach to Care Kit for children (PACK Child). 2019.

22. Arifeen S, Bryce J, Gouws E, Baqui A, Black R, Hoque D, et al. Quality of care for under-fives in firstlevel health facilities in one district of Bangladesh. Bulletin of the World Health Organization. 2005;83:260-7.

23. Baiden F, Owusu-Agyei S, Bawah J, Bruce J, Tivura M, Delmini R, et al. An Evaluation of the Clinical Assessments of Under-Five Febrile Children Presenting to Primary Health Facilities in Rural Ghana. PloS one. 2011;6(12):e28944.

24. Lange $S$, Mwisongo A, Mæstad 0 . Why don't clinicians adhere more consistently to guidelines for the Integrated Management of Childhood IIIness (IMCI)? Social Science \& Medicine. 2014;104:56-63.

25. DeRenzi B, Lesh N, Parikh T, Sims C, Maokla W, Chemba M, et al., editors. E-IMCl: Improving pediatric health care in low-income countries. Proceedings of the SIGCHI conference on human factors in computing systems; 2008: ACM.

26. Coyne I. Families and health-care professionals' perspectives and expectations of family-centred care: hidden expectations and unclear roles. Health Expect. 2015;18(5):796-808.

27. Franke R, Kaud J. The Hawthorne experiments: First statistical interpretation. Am Sociol Rev 1978;43:623-43.

\section{Additional Files}

1. File Name: Additional File 1

File format: Additional file 1.pdf

Title of Data: Characteristics of PACK Child pilot facilities

Description of Data: Brief descriptions of each of the pilot facilities including the total number of staff, the number seeing children, and the average attendance per facility.

2. File Name: Additional File 2

File format: Additional file 2.pdf

Title of Data: Sample of guide

Description of Data: Sample of routine care page of PACK Child guide.

3. File Name: Additional File 3

File format: Additional file 3.pdf 
Title of Data: Summary of Cases

Description of Data: Summary of cases used in the training of PACK Child.

4. File Name: Additional File 4

File format: Additional file 4.pdf

Title of Data: Evolution of PACK Child pilot

Description of Data: A description of the core elements of each phase of the pilot.

5. File Name: Additional File 5

File format: Additional file 5.pdf

Title of Data: Clinician questions coded by type of question and phase of study

Description of Data: Detailed breakdown of focus of consultations and changes across phases.

6. File Name: Additional File 6

File format: Additional file 6.pdf

Title of Data: Proportion of questions about symptoms when routine care delivered/not delivered

Description of Data: Analysis of the proportion of questions asked about symptoms when the routine care page of the guide was used.

\section{Supplementary Files}

This is a list of supplementary files associated with this preprint. Click to download.

- AdditionalFile5.pdf

- AdditionalFile4.pdf

- AdditionalFile2.pdf

- AdditionalFile6.pdf

- AdditionalFile3.pdf

- AdditionalFile1.pdf 